\title{
NOTE STATISTIQUE PRÉLIMINAIRE \\ SUR LES TEIGNES DU MAROC ESPAGNOL
}

\author{
Par M. BAEZA (1)
}

Chargé de mission par la Commission permanente de recherches sanitaires, rattachée à la Direction générale de la Santé en Espagne, j'ai parcouru la zone du protectorat espagnol, au nord du Maroc, dans le but d'étudier les teignes de la population indigène.

Avec l'aide des «Officinas de intervention 》 et de mes confrères médecins, j'ai pu effectuer l'examen systématique des enfants et des adolescents de beaucoup de localités, ainsi que des soldats de quelques unités de troupes indigènes.

Le matériel récolté a été étudié : par examen direct, par montage en préparations permanentes et par cultures. Ce travail a été effectué à Paris, dans la section de mycologie du laboratoire de parasitologie de la Faculté de Médecine, dirigé par le Professeur Brumpt.

Comme résultat de cet examen, je puis donner la statistique préliminaire suivante :

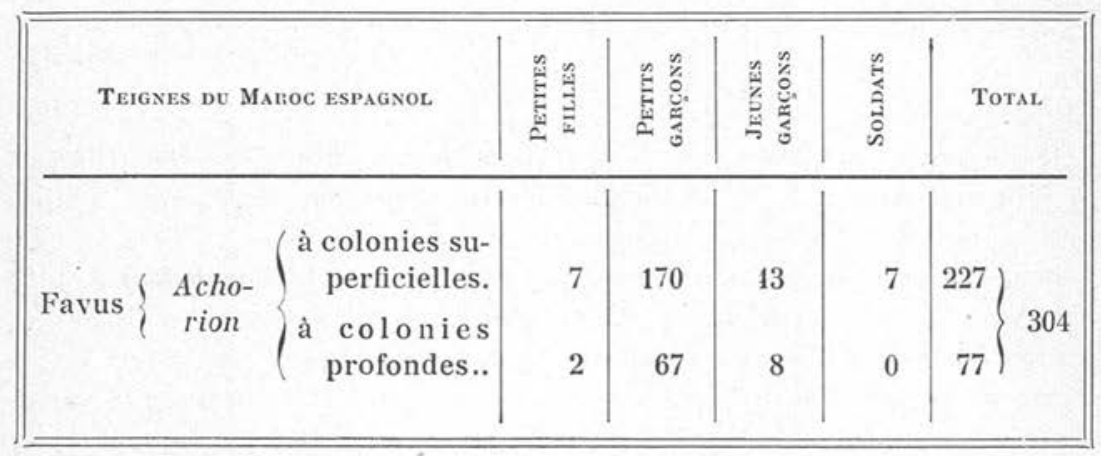

(1) Traduit de l'espagnol par le $\mathrm{D}^{r}$ Maurice LANGeron.

Annales de Parasitologie, T. XII, $\mathbf{N}^{\circ} 5 .-1^{\text {er }}$ septembre 1934, p. $405-407$. 


\begin{tabular}{|c|c|c|c|c|c|c|c|}
\hline \multicolumn{3}{|c|}{ Teignes du Maroc espagnol } & 递 & 总 & 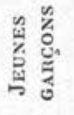 & 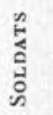 & TотAL \\
\hline \multirow{3}{*}{$\begin{array}{l}0 \\
0 \\
= \\
\vdots \\
0 \\
0 \\
= \\
0 \\
\vdots \\
=\end{array}$} & $\begin{array}{l}\text { Tricho- } \\
\text { phylon } \\
\text { viola- } \\
\text { ceum }\end{array}$ & $\begin{array}{r}\text { colonies bien } \\
\text { pigmentées. } \\
\text { colonies à pig- } \\
\text { ment faible } \\
\text { ou irrégu- } \\
\text { lier........ } \\
\text { colonies non } \\
\text { pigmentées } \\
\text { (ty pe gla- } \\
\text { brum)...... }\end{array}$ & 2 & 72 & 0 & 0 & 49 \\
\hline & \multicolumn{2}{|c|}{$\begin{array}{r}\text { Trichophyton sulphu- } \\
\text { reum................. }\end{array}$} & 0 & 3 & 0 & 0 & 3 \\
\hline & \multicolumn{2}{|c|}{$\begin{array}{c}\text { Infection mixte par } T \text {. } \\
\text { violaceum et } T \text {. sul- } \\
\text { phureum........... }\end{array}$} & 0 & 1 & 0 & 0 & 1 \\
\hline \multicolumn{3}{|c|}{ Total des malades........... } & 12 & 393 & 52 & 7 & 464 \\
\hline \multicolumn{3}{|c|}{ Total des sujets examinés..... } & 257 & \multicolumn{2}{|c|}{1.971 (1) } & 480 & 2.708 \\
\hline \multicolumn{8}{|c|}{$\begin{array}{l}\text { (1) Nous ne pouvons donner séparément le total des deux catégories de } \\
\text { garcons (enfants et adolescents) parce que nous n'avons pu savoir i’âge que } \\
\text { pour les malades. }\end{array}$} \\
\hline
\end{tabular}

Dans cette statistique, il est important de noter : $1^{\circ}$ l'absence $^{\prime}$ de microspories ; $2^{\circ}$ la grande abondance des cas de favus ; $3^{\circ}$ le petit nombre d'espèces parasitaires.

Nous voyons aussi comment varient les caractéristiques parasitologiques de chaque zone et comment on ne peut généraliser à toutes les zones les faits observés dans l'une d'elles.

Si on se contente de prendre les chiffres de cette statistique, sans tenir compte des autres considérations, le nombre des cas de favus paraît, par rapport à celui des cas de trichophyties, plus grand qu'il n'est en réalité, quoi qu'il soit important. La raison en est que, 
parmi les sujets examinés, il y a beaucoup d'adolescents et d'adultes (un grand nombre d'entre eux venaient pour cause de maladie) chez lesquels les trichophyties ont guéri spontanément à la puberté, tandis que le favus persiste. Par contre, les enfants, chez lesquels la trichophytie est plus fréquente que le favus, n'ont pas, par suite des conditions spéciales du milieu (population dispersée et communications difficiles) pu être réunis en assez grand nombre pour que je les examine.

En ce qui concerne le favus, il résulte de mes cultures qu'il faut admettre soit que les godets et plaques typiques, ainsi que l'aspect macroscopique caractéristique du cheveu favique, peuvent être produits par des champignons autres que l'Achorion schönleini, soit qu'il existe plusieurs espèces d'Achorion humains.

Dans cette statistique, je me suis conformé à la théorie classique, en attendant que j'aie achevé un travail actuellement en cours sur les Achorion.

Presque toutes les trichophyties sont dues au Trichophyton violaceum, qui est tantôt très pigmenté, tantôt plus ou moins incolore, avec tous les intermédiaires entre ces deux extrêmes. Cette diversité est si accentuée qu'en considérant des cas isolés on croirait qu'il s'agit d'espèces distinctes.

Section de mycologie du Laboratoire de parasitologie de la Faculté de Médecine de Paris

(Chef de service: $D^{\mathrm{r}} M$. Langeron) 